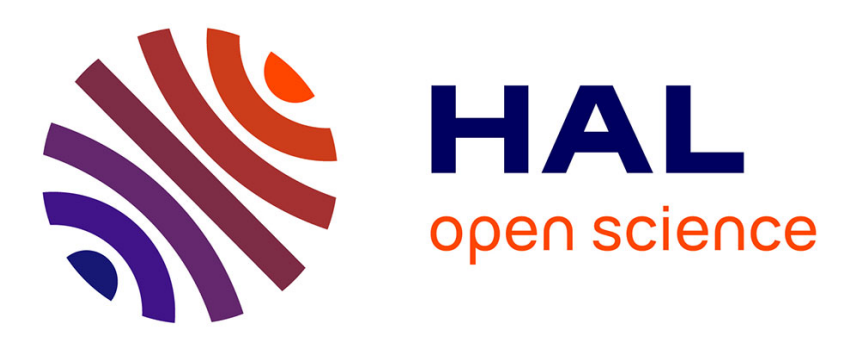

\title{
Étude du signal RPE donné par un spectromètre à hélice court-circuitée en fonction de la fréquence UHF
}

Christian J. Bordé, Pierre Boyer

\section{To cite this version:}

Christian J. Bordé, Pierre Boyer. Étude du signal RPE donné par un spectromètre à hélice courtcircuitée en fonction de la fréquence UHF. Revue de Physique Appliquée, 1967, 2 (2), pp.128-132. 10.1051/rphysap:0196700202012800 . jpa-00242773

\section{HAL Id: jpa-00242773 https://hal.science/jpa-00242773}

Submitted on 1 Jan 1967

HAL is a multi-disciplinary open access archive for the deposit and dissemination of scientific research documents, whether they are published or not. The documents may come from teaching and research institutions in France or abroad, or from public or private research centers.
L'archive ouverte pluridisciplinaire HAL, est destinée au dépôt et à la diffusion de documents scientifiques de niveau recherche, publiés ou non, émanant des établissements d'enseignement et de recherche français ou étrangers, des laboratoires publics ou privés. 


\title{
ÉTUDE DU SIGNAL RPE \\ DONNÉ PAR UN SPEGTROMÈTRE A HÉLICE COURT-GIRGUITÉE EN FONGTION DE LA FRÉQUENGE UHF
}

\author{
Par Ghristian BORdé et Pierre BOYER, \\ Laboratoire de Chimie Théorique, Faculté des Sciences de Nancy, I, rue Grandville.
}

\begin{abstract}
Sommaire. - L'étude est faite en considérant un schéma électrique équivalent, permettant d'appliquer la théorie des lignes.

Dans une première partie, des résultats concernant les spectromètres à cavité sont rappelés puis généralisés dans le cas d'un dispositif électromagnétique quelconque.

Dans une seconde partie, ces résultats sont exploités pour étudier la sensibilité des spectromètres à hélice court-circuitée utilisant un té magique.

Cette étude s'achève par une interprétation de la déformation des signaux RPE lorsqu'on fait varier la fréquence de l'onde UHF.
\end{abstract}

\begin{abstract}
This study is carried out by considering an equivalent circuit and by using the transmission line theory.

In a first part, well known results with cavity spectrometers are recalled and then generalized in the case of any other device.

In a second part, we use the results to study the sensitivity of a shorted helix spectrometer with a magic tee.

In the last part, we explain how the ESR signal changes with the microwave frequency.
\end{abstract}

Introduction. - La cavité résonnante d'un spectromètre à résonance paramagnétique électronique peut être remplacée par une ligne à retard en forme d'hélice. L'une des caractéristiques d'un tel dispositif est d'être à très large bande (contrairement aux cavités). On peut ainsi, d'une manière continue, suivre le signal RPE lorsqu'on fait varier la fréquence de l'onde UHF. On observe alors une déformation continue du signal qui passe périodiquement par des formes d'absorption et de dispersion d'amplitudes très variables. L'étude suivante a pour but de donner une interprétation de ces déformations.

Spectromètre RPE à cavité. - Le schéma électrique équivalent à un système résonnant de pulsation propre $\omega_{\mathrm{r}}$ et de coefficient de surtension à la résonance $Q_{0}=\omega_{\mathrm{r}} P / \Delta P$ est constitué par un circuit oscillant, de paramètres $R, L_{0}, C$ tels que :

$$
\begin{gathered}
L_{0} C \omega_{\mathrm{r}}^{2}=1 \\
L_{0} \omega_{\mathrm{r}} / R=Q_{0} .
\end{gathered}
$$

L'impédance $Z_{0}$ du circuit oscillant équivalent à la cavité vide est donnée par :

$$
Z_{0}=R+j X=R+j\left(L_{0} \omega-\frac{1}{C \omega}\right)
$$

La résonance paramagnétique d'une substance de susceptibilité complexe $\chi=\chi^{\prime}-j \chi^{\prime \prime}$ change $L_{0}$ en :

$$
L=L_{0}\left(1+\eta_{0} \chi\right) \quad \text { (unités MKSA) }
$$

$\eta_{0}$ étant le facteur de remplissage. $Z_{0}$ devient :

$$
Z=Z_{0}+j L_{0} \omega \eta_{0} \chi
$$

Pour la fréquence de résonance du circuit, l'absorption paramagnétique se traduit donc par des variations $\delta R, \delta X, \delta \omega_{\mathrm{r}}, \delta Q_{0}$ telles que :

$$
\begin{aligned}
\delta R / R & =\eta_{0} Q_{0} \chi^{\prime \prime}=-\delta Q_{0} / Q_{0} \\
\delta X / R & =\eta_{0} Q_{0} \chi^{\prime} \\
\delta \omega_{\mathrm{r}} / \omega_{\mathrm{r}} & =-\eta_{0} \chi^{\prime} / 2 .
\end{aligned}
$$

Généralisation. - Si nous voulons pouvoir utiliser un schéma équivalent pour étudier la sensibilité d'un spectromètre où la cavité est remplacée par un dispositif électromagnétique quelconque, il nous faut généraliser les résultats précédents.

Soit $Z=R+j X$ l'impédance du circuit équivalent au dispositif et soit $I$ l'amplitude du courant débité par ce circuit. La quantité $\delta P=\delta R . I^{2} / 2$ représente la puissance moyenne dissipée dans la substance paramagnétique et $\Delta P=R I^{2} / 2$ représente 
la puissance moyenne dissipée par le dispositif électromagnétique.

Par suite :

$$
\frac{\delta R}{R}=\frac{\delta P}{\Delta P}=\frac{Q}{Q_{1}}=\eta Q \chi^{\prime \prime}=-\frac{\delta Q}{Q}
$$

où $Q$ est le facteur de surtention du dispositif défini en fonction des amplitudes $E$ et $B$ du champ électrique et de l'induction magnétique par :

$$
\begin{aligned}
Q=\omega\left(\frac{1}{4 \mu_{0}} \int_{\text {dispositif }}\right. & B^{2} \mathrm{~d} V \\
& \left.+\frac{\varepsilon_{0}}{4} \int_{\text {dispositif }} E^{2} \mathrm{~d} V\right) / \Delta P=\omega \frac{P}{\Delta P}
\end{aligned}
$$

$P$ étant l'énergie moyenne emmagasinée par le dispositif et où $Q_{1}$ est le facteur de surtention moléculaire classique

$$
Q_{1}=1 / \eta \chi^{\prime \prime}
$$

$\eta$ étant un facteur de remplissage défini par :

$$
\begin{aligned}
\eta=\left(\frac{1}{2 \mu_{0}} \int_{\text {substance }} B^{2} \mathrm{~d} V\right) /\left(\frac{1}{4 \mu_{0}} \int_{\text {dispositif }} B^{2} \mathrm{~d} V\right. & \\
& \left.+\frac{\varepsilon_{0}}{4} \int_{\text {dispositif }} E^{2} \mathrm{~d} V\right) .
\end{aligned}
$$

D'autre part, la puissance réactive $\delta X . I^{2} / 2$ est liée à l'énergie magnétique moyenne $\delta W_{\mathbf{M}}$ emmagasinée dans la substance paramagnétique par :

$$
\delta X I^{2} / 2=2 \omega \delta W_{\mathrm{M}} \text {. }
$$

L'énergie $\delta W_{\mathrm{M}}$ est donnée par :

$$
\delta W_{\mathbf{M}}=\frac{\chi^{\prime}}{4 \mu_{0}} \int_{\text {substance }} B^{2} \mathrm{~d} V .
$$

On peut introduire le coefficient de surtension $Q_{2}$ relatif à cette puissance réactive :

$$
Q_{2}=\omega P / 2 \omega \delta W_{\mathrm{M}}=1 / \chi^{\prime} \eta \text {. }
$$

Par suite :

$$
\delta X \mid R=Q / Q_{2}=\eta Q \chi^{\prime} .
$$

Observons que le produit $\eta Q$ peut être défini indépendamment de l'énergie électrique emmagasinée par :

$$
\eta Q=\frac{\omega}{\Delta P} \frac{1}{2 \mu_{0}} \int_{\text {substance }} B^{2} \mathrm{~d} V .
$$

Enfin, si le système présente des fréquences de résonance, on peut aussi généraliser la dernière formule.

Soient $W_{\mathbf{E}}$ et $W_{\mathrm{M}}$ les énergies électrique et magnétique moyennes totales emmagasinées par le dispositif et la substance. La puissance réactive totale s'écrit :

$$
X I^{2} / 2=2 \omega\left[W_{\mathrm{M}}-W_{\mathrm{E}}\right] \text {. }
$$

Une fréquence de résonance est indifféremment définie par :

$$
X=0 \quad \text { ou } \quad W_{\mathrm{M}}=W_{\mathrm{E}} .
$$

REVUE DE PHYSIQUE APPLIQUÉE. - T. 2. No 2. JUIN 1967.
Posons :

avec

$$
\begin{gathered}
W_{\mathrm{M}} / W_{\mathrm{E}}=f\left(\omega, L_{\mathrm{i}}, C_{\mathrm{i}}, R_{\mathrm{i}}\right) \\
f\left(\omega_{\mathrm{r}}, L_{\mathrm{i}}, C_{\mathrm{i}}, R_{\mathrm{i}}\right)=1
\end{gathered}
$$

où les $L_{\mathrm{i}}, C_{\mathrm{i}}, R_{\mathrm{i}}$ sont les valeurs des inductances, capacités et résistances constituant le circuit.

L'absorption paramagnétique se traduit par les variations $\delta L_{\mathrm{i}}, \delta R_{\mathrm{i}}$, des $L_{\mathrm{i}}$ et $R_{\mathrm{i}}$.

$\mathrm{Si}$ on s'était placé à la fréquence de résonance $\omega_{\mathrm{r}}$, cette fréquence devient $\omega_{\mathrm{r}}+\delta \omega_{\mathrm{r}}$ telle que :

$$
f_{\omega}^{\prime} \delta \omega_{\mathrm{r}}+\sum_{\mathrm{i}}\left(f_{L_{\mathrm{i}}}^{\prime} \delta L_{\mathrm{i}}+f_{R_{\mathrm{i}}}^{\prime} \delta R_{\mathrm{i}}\right)=0 .
$$

Pour l'ancienne fréquence $\omega_{\mathrm{r}}$, on a maintenant :

$$
\left(W_{\mathbf{M}}+\delta W_{\mathbf{M}}\right) / W_{\mathrm{E}}=f\left(\omega_{\mathrm{r}}, L_{\mathbf{i}}+\delta L_{\mathbf{i}}, R_{\mathbf{i}}+\delta R_{\mathbf{i}}, C_{\mathbf{i}}\right) .
$$

Soit, puisque $W_{\mathbf{E}} \# W_{\mathbf{M}}$ :

$$
\begin{gathered}
\delta W_{\mathbf{M}}=W_{\mathbf{M}} \cdot \sum_{\mathbf{i}}\left(f_{L_{\mathrm{i}}}^{\prime} \delta L_{\mathbf{i}}+f_{R_{\mathrm{i}}}^{\prime} \delta R_{\mathrm{i}}\right) \\
\delta W_{\mathbf{M}} / W_{\mathbf{M}}=1 / Q_{2}=\eta \chi^{\prime}
\end{gathered}
$$

or

On tire :

$$
f_{\omega}^{\prime}\left(\omega_{\mathbf{r}}\right) \delta \omega_{\mathbf{r}}=-\eta \chi^{\prime} .
$$

Pour un circuit oscillant (résonance parallèle)

$$
f(\omega)=L C \omega^{2} \text {. }
$$

On retrouve bien $\delta \omega_{\mathrm{r}} / \omega_{\mathrm{r}}=-\eta \chi^{\prime} / 2$.

Pour un circuit bouchon sans résistance :

et

$$
\begin{gathered}
f(\omega)=1 / L C \omega^{2} \\
\delta \omega_{\mathbf{r}} / \omega_{\mathbf{r}}=+\eta \chi^{\prime} / 2 .
\end{gathered}
$$

Notons que les fréquences de résonance telles que nous les avons définies ne sont pas forcément celles pour lesquelles le circuit présente une impédance extrêmale.

Spectromètre RPE à hélice. - Le spectromètre à hélice est obtenu en remplaçant la cavité résonnante par une hélice court-circuitée. L'onde UHF incidente et l'onde réfléchie sur le court-circuit créent un système d'ondes stationnaires.

On peut donner une représentation équivalente à l'hélice à l'aide d'une ligne de transmission à constantes distribuées $r, l, C$ (on suppose la conductance transverse nulle), fermée sur une impédance nulle.

La ligne équivalente aura la longueur $L$ de l'hélice et une constante de propagation $\gamma=\alpha+j \beta$ où $\alpha$ est le coefficient d'atténuation de l'hélice et où $\beta=2 \pi / \lambda$, $\lambda$ étant la longueur d'onde apparente de l'onde UHF sur l'hélice. Dans le cas du mode axial pur, on a :

$$
\lambda=\lambda_{0} / 2 \pi n a=\lambda_{0} / S
$$

où $a$ est le rayon de l'hélice, $n$ le nombre de spires par unité de longueur, $\lambda_{0}$ la longueur d'onde dans le vide et $S$ le facteur de ralentissement. 


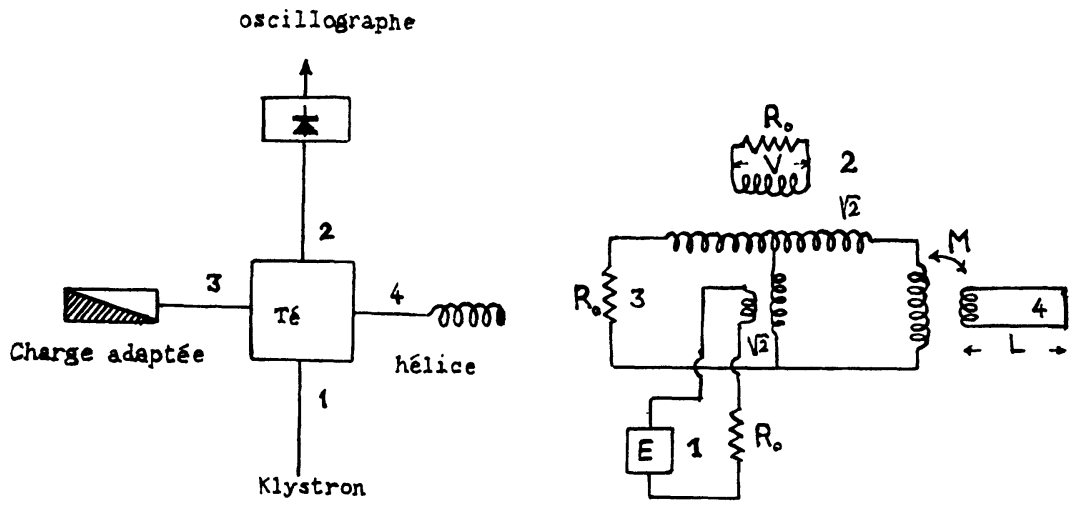

FIG. 1.

(1) $E=$ Force électromotrice équivalente à la puissance délivrée par le klystron de puissance utilisable $E^{2} / 4 R_{0}$.

(2) $V=$ Tension équivalente à la puissance fournie au cristal.

(3) Charge adaptée présentant l'impédance caractéristique $R_{0}$ du guide.

(4) Hélice de longueur $L$. Couplage avec le té magique avec un coefficient d'inductance mutuelle $M$.

L’impédance d'entrée de la ligne est donnée par [1] :

$$
Z=Z_{0} \text { th } \gamma L
$$

où $Z_{0}$ est l'impédance caractéristique de la ligne. Ramenée au primaire du transformateur de coefficient d'inductance mutuelle $M$, elle donne l'impédance couplée :

$$
Z_{\mathrm{R}}=\omega^{2} M^{2} / Z_{0} \text { th } \gamma L .
$$

On en déduit le coefficient de réflexion du guide d'impédance $R_{0}$ fermé sur l'impédance $Z_{\mathrm{R}}$

$$
\Gamma=\frac{Z_{\mathrm{R}}-R_{0}}{R_{0}+Z_{\mathrm{R}}}
$$

et la tension $V$ équivalente à la puissance détectée par le cristal :

$$
\begin{aligned}
& V=\frac{E \Gamma}{4}=\frac{E}{4} \frac{\frac{\omega^{2} M^{2}}{R_{0}}-Z_{0} \text { th } \gamma L}{\frac{\omega^{2} M^{2}}{R_{0}}+Z_{0} \text { th } \gamma L} \\
&=\frac{E}{4} \frac{\frac{\omega^{2} M^{2}}{R_{0}}-R-j X}{\frac{\omega^{2} M^{2}}{R_{0}}+R+j X}(\mathbf{1})
\end{aligned}
$$

où on a posé :

$$
\begin{aligned}
& R=Z_{0} \frac{\operatorname{sh} \alpha L \operatorname{ch} \alpha L}{\operatorname{ch}^{2} \alpha L \cos ^{2} \beta L+\operatorname{sh}^{2} \alpha L \sin ^{2} \beta L} \\
& \text { et } \quad X=Z_{0} \frac{\sin \beta L \cos \beta L}{\operatorname{ch}^{2} \alpha L \cos ^{2} \beta L+\operatorname{sh}^{2} \alpha L \sin ^{2} \beta L} .
\end{aligned}
$$

Observons tout d'abord que si on suppose $r \ll \omega L$

$$
Z_{\mathbf{0}}=\sqrt{\frac{l}{C}}\left[1-j \frac{r}{2 \omega l}\right] \# \sqrt{\frac{l}{G}} .
$$

(1) En remplaçant $Z_{0}$ par $R_{0}$, on retrouve l'expression donnée par Wilmshurst, Gambling et Ingram [2].
Par suite, $R$ et $X$ sont des quantités réelles représentant donc respectivement la résistance et la réactance de la ligne. La sensibilité du spectromètre est représentée par :

$$
\delta V=(\partial V / \partial R)_{X} \delta R+(\partial V / \partial X)_{R} \delta X
$$

où les variations $\delta R$ et $\delta X$ de $R$ et $X$ dues à l'absorption paramagnétique sont liées à $\chi^{\prime}$ et $\chi^{\prime \prime}$ par les relations démontrées précédemment.

De l'expression de $V$, on déduit :

$$
\begin{aligned}
\delta V=- & \frac{E}{2} \frac{\omega^{2} M^{2} / R_{0}}{\left(\frac{\omega^{2} M^{2}}{R_{0}}+R+j X\right)^{2}}(\delta R+j \delta X) \\
& =-\eta Q \frac{E}{2} \frac{\omega^{2} M^{2} / R_{0}}{\left(\frac{\omega^{2} M^{2}}{R_{0}}+R+j X\right)^{2}}\left(\chi^{\prime \prime}+j \chi^{\prime}\right)
\end{aligned}
$$

où $\eta Q$ varie peu avec la fréquence $\nu$ de l'onde UHF.

L'optimisation de l'adaptation guide-hélice donne :

$$
\left(\omega^{2} M^{2} / R_{0}\right)^{2}=R^{2}-X^{2} .
$$

$\delta V$ devient :

$$
\delta V=-\eta Q \frac{E}{2} \frac{\sqrt{1-\left(X^{2} / R^{2}\right)}}{\left[1+\sqrt{1-\left(X^{2} / R^{2}\right)}+(j X / R)\right]^{2}}\left(\chi^{\prime \prime}+j \chi^{\prime}\right) .
$$

La sensibilité maximum du spectromètre correspondra donc aux résonances et antirésonances de la ligne, soit :

10 Pour les résonances :

$$
\begin{aligned}
L & =k \lambda / 2 \quad k \text { entier } \\
R & =Z_{0} \text { th } \alpha L \# Z_{0} \alpha L=k \frac{\pi}{2} \frac{Z_{0}}{Q_{0}} \\
X & =0
\end{aligned}
$$

où $Q_{0}$ est le facteur de surtension de la ligne

$$
Q_{0}=l \omega / r=\beta / 2 \alpha \text {. }
$$


Sachant que $\alpha=(r / 2) \sqrt{l / C}$ et $\beta=\omega \sqrt{l \bar{C}}$, on tire :

$$
Q_{0}=\pi S v / \alpha C_{0}
$$

où $C_{0}$ est la vitesse de la lumière dans le vide et avec $S=2 \pi n a$.

Chaque fois que $X=0$, c'est-à-dire pour les résonances, et comme nous allons le voir pour les antirésonances, l'énergie magnétique moyenne emmagasinée est égale à l'énergie électrique moyenne emmagasinée. Il en résulte que pour les fréquences correspondantes le coefficient de surtension de la ligne $Q_{0}=l \omega / r$ est identique au coefficient $Q=\omega P / \Delta P$ précédemment défini et que

$$
\eta=\eta_{0}=\int_{\text {substance }} B^{2} \mathrm{~d} V / \int_{\text {hélice + extérieur }} B^{2} \mathrm{~d} V
$$

$2^{\circ}$ Pour les antirésonances :

$$
\begin{aligned}
L & =(2 k+1) \lambda / 4 \\
R & =Z_{0} \operatorname{coth} \alpha L \# \frac{Z_{0}}{\alpha L}=Z_{0} \frac{4}{\pi} \frac{Q_{0}}{2 k+1} \\
X & =0 .
\end{aligned}
$$

Observons que $R$ est alors très grand (nous supposerons toujours $\alpha L \ll 1$ ). La sensibilité n'est donc grande pour les antirésonances que si $\omega^{2} M^{2} / R_{0}$ peut être rendu assez grand pour que la condition d'adaptation soit satisfaite.

Si $\omega^{2} M^{2} / R_{0}$ est très inférieur à $Z_{0} / \alpha L$, la sensibilité est au contraire minimum pour les antirésonances de la ligne.

Pour une fréquence de résonance (ou d'antirésonance), la condition d'adaptation s'écrit $\left(^{2}\right)$ :

$$
\begin{array}{cc} 
& R=\omega^{2} M^{2} / R_{0} \text { par suite } V=0 \\
\text { et } & |\delta V|=(E / 8) \eta_{0} Q_{0}\left(\chi^{\prime \prime}+j \chi^{\prime}\right) .
\end{array}
$$

On obtient donc un mélange de signaux d'absorption et de dispersion.

En fait, $V$ n'est nul que si le té est parfaitement équilibré. Suivant la phase de $V$ par rapport à $R$ et $X$, c'est soit $(\partial V / \partial R)_{X}$, soit $(\partial V / \partial X)_{R}$ qui donne un effet du premier ordre sur le module de $V$.

$\mathrm{A}$ la résonance et à l'antirésonance, si, par suite d'une mauvaise adaptation, $V$ est différent de zéro, on voit sur l'expression donnant $V$ que $V$ est réel, c'est-àdire qu'on observera un signal d'absorption (ceci suppose $V$ suffisamment grand devant $\delta V$ pour que la détection soit linéaire en $\delta V$ ).

D'autre part, on monte généralement dans le bras 3 un pont comportant un déphaseur et un atténuateur.

Si $Z^{\prime}$ est l'impédance ainsi introduite, $V$ devient :

$$
V_{\text {tot }}=\frac{E}{4} \frac{R_{0}-Z^{\prime}}{Z^{\prime}+R_{0}}+\frac{E}{4} \frac{Z_{\mathrm{R}}-R_{0}}{R_{0}+Z_{\mathrm{R}}}=V^{\prime}+V
$$

Cette opération ne change rien à la valeur de $\delta V$ et, suivant la phase de $V^{\prime}+V$, on observera à volonté

(2) $\mathrm{Si}$ au lieu d'utiliser le coefficient d'induction mutuelle $M$, on introduit le rapport de transformation $N$, la résistance ramenée $\omega^{2} M^{2} / R_{0}$ s'écrit $N^{2} R$ et la condition d'adaptation devient $R_{0}=N^{2} R$. un signal d'absorption ou de dispersion. En prenant $V^{\prime} \gg \delta V$, ceci assure la linéarité de la détection.

Dans le cas d'un signal d'absorption, il est classique de comparer celui-ci au signal de bruit thermique.

$\mathrm{Si}$ le détecteur a un facteur de bruit $f$ et une largeur de bande $B$, le signal RPE sera égal au bruit thermique pour :

$$
\delta V=\left(f k T B R_{0}\right)^{1 / 2}
$$

d'où la valeur minimum de $\chi^{\prime \prime}$ :

$$
\chi_{\min .}^{\prime \prime}=4(f k T B)^{1 / 2} / P^{1 / 2} \eta_{0} Q_{0}
$$

où $P=E^{2} / 4 R_{0}$ est la puissance utilisable délivrée par le klystron.

Enfin, cherchons quelle est la variation $\delta \omega_{\mathrm{r}}$ des pulsations de résonance et d'antirésonance de la ligne.

Pour une fréquence de résonance $\omega_{\mathrm{r}}$ définie par

$$
\beta_{\mathrm{r}}=\omega_{\mathrm{r}} \sqrt{l \bar{l}}=k \frac{\pi}{L}
$$

on peut écrire :

$R_{0} \# Z_{0} \alpha L \quad$ et $\quad \delta X \# Z_{0} L \delta \beta_{\mathrm{r}}=Z_{0} L \omega_{\mathrm{r}} \delta l / 2 \sqrt{l \overline{l C}}$.

La fréquence de résonance devient $\omega_{\mathrm{r}}+\delta \omega_{\mathrm{r}}$ telle que :

$$
\sqrt{\overline{l C}} \delta \omega_{\mathrm{r}}+\frac{\omega_{\mathrm{r}}}{2 \sqrt{l C}} \delta l=0
$$

d'où :

$$
\frac{\delta \omega_{\mathrm{r}}}{\omega_{\mathrm{r}}}=-\frac{\delta l}{2 l C}=-\frac{\delta X}{R} \frac{\alpha}{\beta_{\mathrm{r}}}=-\frac{\delta X}{R} \cdot \frac{1}{2 Q_{0}}
$$

soit : $\quad \delta \omega_{\mathrm{r}} / \omega_{\mathrm{r}}=-\eta \chi^{\prime} / 2$

formule identique à celle obtenue pour un circuit oscillant. Pour une fréquence d'antirésonance

$$
R_{0} \# Z_{0} / \alpha L \text { et } \delta X \#-Z_{0} L \delta \beta_{\mathrm{r}} / \alpha^{2} L^{2} \text {. }
$$

Le même calcul donne :

$$
\delta \omega_{\mathrm{r}} / \omega_{\mathrm{r}}=+\eta \chi^{\prime} / 2
$$

formule identique à celle obtenue pour un circuit bouchon.

Vérifions, dans le cas de la ligne court-circuitée, la formule générale démontrée précédemment. En faisant le rapport des intégrales des énergies magnétique et électrique moyennes emmagasinées sur la longueur de la ligne, on obtient facilement :

$$
f(\omega)=\frac{W_{\mathrm{M}}}{W_{\mathrm{E}}}=\frac{\omega \sqrt{l C} \operatorname{sh} 2 \alpha L+\alpha \sin 2 \omega \sqrt{l C} L}{\omega \sqrt{l C} \operatorname{sh} 2 \alpha L-\alpha \sin 2 \omega \sqrt{l C} L}
$$

d'où on tire :

$$
\begin{aligned}
f^{\prime}\left(\omega=\omega_{\mathrm{r}}\right)=\frac{2}{\omega_{\mathrm{r}}} \cos 2 \omega_{\mathrm{r}} L \\
\quad=\left\{\begin{array}{l}
+\frac{2}{\omega_{\mathrm{r}}} \text { pour les résonances } \\
-\frac{2}{\omega_{\mathrm{r}}} \text { pour les antirésonances }
\end{array}\right.
\end{aligned}
$$

et on retrouve bien les deux formules précédentes. 
Déformation du signal RPE avec la fréquence. Le spectromètre RPE à hélice permet d'obtenir un signal dans une très large bande de fréquence $\left(^{3}\right)$.

Lorsqu'on fait varier la fréquence UHF (en maintenant le balayage du champ magnétique autour de la valeur correspondante), le signal se déforme de manière continue et passe alternativement par des formes d'absorption et de dispersion [3].

Les calculs précédents fournissent la longueur optimale de l'hélice et la condition d'adaptation pour une fréquence donnée. Inversement, si $L$ et $M$ sont constants et si la fréquence varie, les extremums d'amplitude du signal correspondront à $L=k \lambda / 2$ et $L=(2 k+1) \lambda / 4$.

Pour interpréter les déformations du signal lorsque $L$ et $M$ sont constants, écrivons $\delta V$ sous la forme :

$$
\delta V=\eta Q R(\partial V / \partial R)_{X}\left(\chi^{\prime \prime}+j \chi^{\prime}\right)=\delta V_{1}+\delta V_{2}
$$

avec

$$
\text { et } \quad \delta V_{2}=j \eta Q R(\partial V / \partial R)_{X} \chi^{\prime} \text {. }
$$

D'autre part, $V$ s'exprime en fonction de $(\partial V / \partial R)_{X}$ par :

$\mathrm{V}_{\mathrm{tot}}=-\frac{x}{1+x}\left(\frac{\partial V}{\partial R}\right)_{X}\left[\frac{\omega^{2} M^{2}}{R_{0} x}-\frac{R^{2}-X^{2}}{\omega^{2} M^{2} / R_{0}}\right.$

$$
\left.+R \frac{1-x}{x}-2 \frac{j R X}{\omega^{2} M^{2} / R_{0}}+j X \frac{1-x}{x}\right]
$$

où

$$
x=Z^{\prime} \mid R_{0}
$$

( $\left.{ }^{3}\right)$ Si la valeur de $M^{2} \omega^{2}$ est assez grande devant $Z_{0} \alpha L$, on pourra suivre le signal partout sans retoucher à l'adaptation. $\delta V=\delta V_{1}+\delta V_{2}$ est la somme d'une contribution due à l'absorption et d'une contribution due à la dispersion.

La phase relative de $V$ et de chacune de ces contributions dépendra de la phase relative de $V$ et $(\partial V / \partial R)_{X}$.

Supposons $x$ réel pour avoir un signal d'absorption à la résonance et à l'antirésonance.

Lorsque $|X|$ augmente de part et d'autre de la résonance, il se superpose au signal d'absorption un signal de dispersion positif ou négatif suivant le signe de $X$ et de $\left(\frac{1-x}{x}-\frac{2 R}{\omega^{2} M^{2} / R_{0}}\right)$, puis ce signal s'annule (seulement si $x<1$ ) à nouveau pour :

$$
\frac{1-x}{x}=\frac{2 R}{\omega^{2} M^{2} / R_{0}} \text {. }
$$

Il réapparaît au-delà de cette valeur pour subsister seul lorsque :

$$
\frac{R^{2}}{\omega^{2} M^{2} / R_{0}}=\frac{X^{2}}{\omega^{2} M^{2} / R_{0}}+R \frac{1-x}{x}+\frac{\omega^{2} M^{2}}{R_{0} x} .
$$

Un nouveau signal d'absorption de sens opposé à celui observé pour la résonance se superpose et subsiste seul à l'antirésonance.

Cette théorie permet de justifier la déformation continue du signal RPE lorsque la fréquence de l'onde UHF varie. Elle montre d'autre part la nécessité d'utiliser un système $\mathrm{AFG}$ pour une hélice résonnante. En effet, si $\chi^{\prime}$ varie entre des valeurs trop élevées au voisinage de la résonance paramagnétique, la fréquence de résonance de la ligne variera beaucoup au cours du balayage et le signal sera déformé.

Manuscrit reçu le 21 septembre 1966.

\section{BIBLIOGRAPHIE}

[1] Stater (J. C.), Microwave Transmission, Dover Publications, New York, 1959.

[2] WIIMShuRsT (T. H.), GAmbling (W. A.), INGRAM (D. J. F.), J. Electronics and Control, oct. 1962, 13, 339 .

[3] BordÉ (Ch.), Dipl. Études Sup., Nancy, 1966. 